Original Article

\title{
ADULT BASIC LIFE SUPPORT (BLS) AWARENESS AND KNOWLEDGE AMONG MEDICAL AND DENTAL INTERNS COMPLETING INTERNSHIP FROM DEEM ED UNIVERSITY
}

\author{
Raghava Sharma ${ }^{1}$, Nazir R. Attar ${ }^{2}$ \\ ${ }^{1}$ Professor, ${ }^{2}$ Professor, Department of Medicine, K.S. Hegde M edical Acadamy, Deralakatte, M angalore - 575018. \\ Correspondence: \\ Raghava Sharma \\ Professor, Department of M edicine, K.S. Hegde M edical Acadamy, Deralakatte, M angalore - 575018. \\ Mobile : 09448770919 E-mail : rrsharma1967@yahoo.com
}

\begin{abstract}
:
The present study was aimed to assess the awareness, knowledge, and attitude towards basic life support (BLS) among the interns completing their internship from both medical and dental streams of the Nitte university (a deemed university at M angalore, Karnataka) and also to identify the areas to be addressed for improving the standards of BLS among the interns at their crucial juncture of moving out to the community, society as health care providers.
\end{abstract}

A descriptive study was conducted by using a Questionnaire comprising of 19 questions to collect the data pertaining to demographic details, awareness and knowledge of BLS, attitude towards BLS among all the medical and dental interns completing their internship during March 2012. Thestudy was conducted in the last week of their one year internship programme.

After excluding the incomplete response sheets which were none in the present study, the data from 162 interns were subjected to the analysis. The M ain outcome measure was the over all score in the BLS knowledge. Knowledge of BLS was assessed as per the data contained in the Basic life support manual from American Heart Association.

The results were drawn based on the comparisons between M edical and Dental streams and also with in each stream. Out of 162 interns, 84 were medical interns and 78 were dental interns. All of them (100\%) were aware of the BLS and its usefulness. $16(19 \%)$ medical interns had complete knowledge of BLS while none (0\%) among dental interns had complete knowledge of BLS. A score of less than $50 \%$ was evident in $37(44 \%)$ of medical interns and $69(88 \%)$ of dental interns thus indicating a poor knowledge of BLS among both medical and dental interns who were completing their internship. However medical interns scored better in comparison to dental interns. Resuscitation experience (performing BLS) and Training (attending BLS work shop) resulted in better BLS knowledge and better scoring pattern among the medical interns thus boosting the confidence among interns. (Statistically significant with $\mathrm{P}<0.05$ ).

Present study highlightsthe need for a structured training of BLS and inclusion of BLSin the Medical and Dental academic curriculum.

Keywords: •Basic life support $\bullet$ BLS awareness $\bullet$ BLS knowledge $\bullet$ M edical, Dental interns completing internship

\section{Introduction :}

Cardiac arrest is an important acute emergency situation both with in and out side the hospital set ups and carries a high level of mortality risk. However if early Basic life support (BLS) - cardio pulmonary resuscitation (CPR) is

\begin{tabular}{|c|}
\hline Access this article online \\
\hline Quick Response Code \\
\hline \\
\hline
\end{tabular}
initiated; the survival rate can be substantially improved ${ }^{1}$ The knowledge of BLS (CPR) is a major determinant in the success of resuscitation and plays a vital role in the final out come of acute emergency situations. Knowledge of BLS is an absolute necessity for medical professionals to face acute medical emergencies. In the present study we aimed to assess the awareness, knowledge, attitude about adult BLS among interns completing their internship in both medical and dental streams from Nitte University (a deemed university).

\section{Relevance and need for the present study:} Chandrasekaran S and group studied the awareness of BLS among students, doctors, and nurses of medical, dental, homeopathy $\&$ nursing colleges and found it to be very poor which needed improvement ${ }^{2}$ 
S Raghavan and group from resuscitation council of southern Africa analyzed the level of knowledge $\&$ skill in basic resuscitation among medical practitioners (full time employed doctors) and tried to determine the differences in characteristics between those with and without knowledge and skills. In this cross sectional study they also found poor knowledge and skills of basic resuscitation among medical practitioners as less than $25 \%$ of them scored more than $50 \%$ marks $^{3}$

Hassan Zaheer \& group from Karachi ,Pakistan analyzed under graduate medical students and found around $60 \%$ of them had no knowledge of BLS and concluded that inclusion of BLS course will increase awareness and application of this valuable life saving maneuver ${ }^{4}$.

PS Phillips and group from Royal hospital Bath (UK) analyzed the junior doctors in medical schools of $\mathrm{U} K$ and found many junior doctors were not competent in carrying out effective resuscitation even though medical schools in U K provided them some form of life support training ${ }^{5}$.

Ji Ung $\mathrm{Na}$ and group from Korea investigated the level of BLS skill retention of Medical Doctors six and twelve months after BLS education and analyzed the correlation between Clinical experience of resuscitation and BLS skill retention. They found well Preserved Compression skills but poor retention of non compression skills ${ }^{6}$.

The above literatures indicate that BLS studies have been conducted in various Countries on specific sub groups like under graduate medical and dental students, medical doctors in medical colleges, full time employed doctors in government hospitals, junior doctors in medical schools.

HOWEVER THERE ARE NO STUDIES TO KNOW THE BLS PREPAREDNESS AMONG MEDICALAND DENTALINTERNS RIGHT AT THE TIME OF COM PLETING THEIR INTERNSHIP, A CRUCIAL TIME PERIOD JUST BEFORE THEY REACH OUT TO THECOM M UNITY ASHEALTH CAREPROVIDERS.

Hence the present study aims to know the level of BLS preparedness among this particular sub group and secondly it shall also help the administrators to streamline necessary modalities to make our future health care providersfull fledged.

\section{Methods And Methodology:}

The present descriptive study was conducted in a Questionnaire format by using a Questionnaire containing 19 questions pertaining to the demographic details, awareness, knowledge, attitude towards BLS. The protocol was approved by the central ethics committee of Nitte University. Whole batch of medical interns $(n=84)$ and dental interns $(n=78)$ who were Completing their internship were included in the study to avoid any selection bias.

After obtaining the informed consent from each participant, each one was asked to fill up the provided questionnaire (Appendix II) in front of the investigator to avoid any malpractice while answering the questionnaire. The answer keys for the core questions on knowledge of BLS were generated using Basic life support manual from American Heart Association. Incomplete response sheets (none in the present study) were excluded from data capturing and analysis. The data from 162 interns so captured were subjected to both descriptive and inferential statistical analysis. STATISTICAL ANALYSIS: The statistical analysis was performed with the statistical Package for the social sciences, Windows version 11.0 (SPSS Inc, Chicago, US). As the whole batches of interns completing internship were included (none excluded) the Sample thus constituted represented the Population, eliminating the scope for Standard error. Associations with in the medical $\&$ dental streams and comparisons between medical and dental streams were drawn using chi square and $\mathrm{z}$ test. The results with a $\mathrm{P}$ value of $<0.05$ were considered significant. The conclusions were drawn based on the results of the analysis. 
Results :

Table - 1 (Demographic Details)

\begin{tabular}{|l|c|c|}
\hline PARAMETER & MEDICAL INTERNS $(\mathbf{n}=84)$ & DENTAL INTERNS $\mathbf{n}=\mathbf{7 8})$ \\
\hline $\begin{array}{l}\text { - Total Number of participant (interns } \\
\text { in the study) }\end{array}$ & 84 & 78 \\
\hline - Sex (F / M ) & & $(12 / 66)$ \\
\hline - AGE & $(37 / 47)$ & $(15 \% / 85 \%)$ \\
\hline
\end{tabular}

Table - 2 (Awareness and attitude towards BLS)

\begin{tabular}{|l|c|c|}
\hline PARAMETERS & $\begin{array}{c}\text { MEDICAL INTERNS } \\
(\mathbf{n}=\mathbf{8 4})\end{array}$ & $\begin{array}{c}\text { DENTAL INTERNS } \\
(\mathbf{n}=\mathbf{7 8})\end{array}$ \\
\hline${ }^{*}$ Able to expand the term BLS & $84(100 \%)$ & $78(100 \%)$ \\
\hline $\begin{array}{l}* \text { Aware of the necessity \& usefulness of } \\
\text { knowing BLS }\end{array}$ & $84(100 \%)$ & $78(100 \%)$ \\
\hline $\begin{array}{l}* \text { Recommended BLS inclusion in } \\
\text { academic curriculum }\end{array}$ & $84(100 \%)$ & $78(100 \%)$ \\
\hline
\end{tabular}

Table - 3 (Knowledge of BLS)

\begin{tabular}{|l|c|c|}
\hline PARAMETERS & $\begin{array}{c}\text { MEDICAL INTERNS } \\
(\mathbf{n}=\mathbf{8 4})\end{array}$ & $\begin{array}{c}\text { DENTAL INTERNS } \\
(\mathbf{n}=\mathbf{7 8})\end{array}$ \\
\hline - Knowledge of set ups where & & \\
BLS can be performed: & $11(13 \%)$ & $15(19 \%)$ \\
$*$ In only hospital set up & $73(87 \%)$ & $63(81 \%)$ \\
* Both with in \& out side hospital set up & $82(98 \%)$ & $41(53 \%)$ \\
\hline - Observed BLS being Performed: & $02(2 \%)$ & $37(47 \%)$ \\
$*$ Yes & & \\
$*$ No & $71(85 \%)$ & $0(0 \%)$ \\
\hline - Performed BLS by self : & $13(15 \%)$ & $78(100 \%)$ \\
$*$ Yes & $71(85 \%)$ & $6(8 \%)$ \\
$*$ No & $13(15 \%)$ & $72(92 \%)$ \\
\hline - Obtained prior BLS training at work shops: & & \\
$*$ Yes & No &
\end{tabular}

Table - 4 (Knowledge of individual components of BLS)

\begin{tabular}{|l|c|c|}
\hline PARAMETERS & $\begin{array}{c}\text { MEDICAL INTERNS } \\
(\mathbf{n}=\mathbf{8 4})\end{array}$ & $\begin{array}{c}\text { DENTAL INTERNS } \\
\text { ( } \mathbf{n}=\mathbf{7 8})\end{array}$ \\
\hline $\begin{array}{l}\text { - Knowledge of rate of external cardiac } \\
\text { massage per minute during BLS: }\end{array}$ & & \\
* Knows & $61(73 \%)$ & $57(73 \%)$ \\
$*$ Does not know & $23(27 \%)$ & \\
\hline - Knowledge of ratio of cardiac compress- & & $38(49 \%)$ \\
ions to breaths delivered during BLS: & $70(83 \%)$ & $40(51 \%)$ \\
$*$ Knows & $14(17 \%)$ & \\
$*$ Does not know & & $25(32 \%)$ \\
\hline - Knowledge of location For chest & $65(77 \%)$ & \\
compression while delivering BLS: & $19(23 \%)$ & \\
$*$ correct location & & $10(13 \%)$ \\
$*$ wrong location & $21(25 \%)$ & $68(87 \%)$ \\
\hline - Knowledge of sequence to be followed & $63(75 \%)$ & \\
while performing BLS: & & \\
$*$ correct sequence & & \\
$*$ Wrong sequence & & \\
\hline
\end{tabular}


Table - 5 (Scores scored \& self grading of BLS knowledge)

\begin{tabular}{|l|c|c|}
\hline SCORES & $\begin{array}{c}\text { MEDICAL INTERNS } \\
(\mathbf{n}=\mathbf{8 4})\end{array}$ & $\begin{array}{c}\text { DENTAL INTERNS } \\
(\mathbf{n}=\mathbf{7 8})\end{array}$ \\
\hline - Scores scored by interns : & $01(1 \%)$ & \\
a. $0 \%$ & $15(18 \%)$ & $08(10 \%)$ \\
b. $25 \%$ & $21(25 \%)$ & $20(26 \%)$ \\
c. $50 \%$ & $31(37 \%)$ & $41(52 \%)$ \\
d. $75 \%$ & $16(19 \%)$ & $09(12 \%)$ \\
e. $100 \%$ & & $00(0 \%)$ \\
\hline - Self assessment of reasons for their lack & & \\
of BLS knowledge : & $56(67 \%)$ & $58(75 \%)$ \\
a. Non availability of professional training & $09(11 \%)$ & $07(9 \%)$ \\
b. Lack of interest & $08(10 \%)$ & $08(10 \%)$ \\
c. Busy curriculum & $11(13 \%)$ & $05(6 \%)$ \\
d. Various combinations of above 3 factors & & \\
\hline - Self grading of BLS Knowledge level: & $03(4 \%)$ & $20(26 \%)$ \\
a. Poor & $07(8 \%)$ & $17(22 \%)$ \\
b. Below average & $52(62 \%)$ & $39(50 \%)$ \\
c. Average & $18(21 \%)$ & $02(2 \%)$ \\
d. Good & $04(5 \%)$ & $00(0 \%)$ \\
e. Excellent &
\end{tabular}

Table - 6 (Individual BLS knowledge components \& associations in medical interns)

\begin{tabular}{|c|c|c|c|c|c|c|c|c|}
\hline \multirow{4}{*}{$\begin{array}{l}\text { MEDICAL } \\
\text { INTERNS } \\
\text { - } \text { Rate knowledge } \\
\text { YES } \\
\text { NO }\end{array}$} & \multicolumn{2}{|c|}{$\begin{array}{l}\text { BLS Work shop./ } \\
\text { training Attended } \\
\quad(n=71)\end{array}$} & \multicolumn{2}{|c|}{$\begin{array}{c}\text { BLS Work shop / } \\
\text { training not attended } \\
(\mathrm{n}=13)\end{array}$} & \multicolumn{2}{|c|}{$\begin{array}{l}\text { BLS Performed / } \\
\text { BLS experience } \\
(n=71)\end{array}$} & \multicolumn{2}{|c|}{$\begin{array}{l}\text { BLS not performed } \\
\qquad(n=13)\end{array}$} \\
\hline & (0) & (E) & (0) & (E) & (0) & (E) & (0) & (E) \\
\hline & 55 & 51.56 & 06 & 9.44 & 55 & 51.56 & 06 & 9.44 \\
\hline & 16 & 19.44 & 07 & 3.56 & 16 & 19.44 & 07 & 3.56 \\
\hline - Ratio Knowledge & & & & & & & & \\
\hline YES & 58 & 59.16 & 12 & 10.83 & 60 & 59.16 & 10 & 10.83 \\
\hline NO & 13 & 11.83 & 01 & 2.16 & 11 & 11.83 & 03 & 2.16 \\
\hline $\begin{array}{l}\text { - Location } \\
\text { Knowledge }\end{array}$ & & & & & & & & \\
\hline YES & 58 & 54.94 & 07 & 10.06 & 60 & 54.94 & 05 & 10.06 \\
\hline NO & 13 & 16.06 & 06 & 2.94 & 11 & 16.06 & 08 & 2.94 \\
\hline $\begin{array}{l}\text { - Sequence } \\
\text { Knowledge }\end{array}$ & & & & & & & & \\
\hline YES & 20 & 17.75 & 01 & 3.25 & 19 & 17.75 & 02 & 3.25 \\
\hline NO & 51 & 53.25 & 12 & 9.75 & 52 & 53.25 & 11 & 9.75 \\
\hline
\end{tabular}

Table - 7 (Individual BLS knowledge components and associations in dental interns)

\begin{tabular}{|ll|c|c|}
\hline DENTAL INTERNS & $\begin{array}{c}\text { BLS Work shop / BLS } \\
\text { training attended ( } \mathbf{n = 6})\end{array}$ & $\begin{array}{c}\text { BLS Performed / } \\
\text { BLS experience }(\mathbf{n}=\mathbf{0})\end{array}$ \\
\hline - Rate knowledge & Yes & $03(50 \%)$ & $\begin{array}{c}\text { As none of the Dental } \\
\text { interns had performed }\end{array}$ \\
\hline - Ro & $03(50 \%)$ & BLS, the relationship \\
& Yes & $03(50 \%)$ & association between \\
\hline - Location knowledge & Yes & $03(50 \%)$ & performing BLS and \\
& No & $00(0 \%)$ & individual BLS knowledge \\
\hline - Sequence knowledge & Yes & $06(100 \%)$ & components not analyzed. \\
& No & $00(0 \%)$ & \\
\hline
\end{tabular}


Table - 8 (Association between Total BLS knowledge, BLS training,

BLS experience among M edical interns )

\begin{tabular}{|c|c|c|c|c|}
\hline $\begin{array}{l}\text { Medical interns } \\
\text { BLS knowledge } \\
\text { Scores Scored }\end{array}$ & $\begin{array}{l}\text { BLS Work shop./ } \\
\text { training Attended } \\
\text { (n=71) }\end{array}$ & $\begin{array}{c}\text { BLS Work shop / } \\
\text { training not attended } \\
(\mathrm{n}=13)\end{array}$ & $\begin{array}{c}\text { BLS Performed / } \\
\text { BLS experience } \\
(n=71)\end{array}$ & $\begin{array}{l}\text { BLS not performed } \\
\qquad(n=13)\end{array}$ \\
\hline \multicolumn{5}{|l|}{ MEDICAL INTERNS } \\
\hline $0 \%$ & $01(\mathrm{e}=0.833)$ & $00(e=0.167)$ & $00(e=0.845)$ & $01(e=0.155)$ \\
\hline $25 \%$ & $09(\mathrm{e}=12.15)$ & $06(e=2.5)$ & $08(\mathrm{e}=12.679)$ & $07(\mathrm{e}=2.321)$ \\
\hline $50 \%$ & $18(\mathrm{e}=17.5)$ & $03(e=3.5)$ & $20(e=17.75)$ & $01(\mathrm{e}=3.25)$ \\
\hline $75 \%$ & $26(e=25.83)$ & $05(e=5.17)$ & $29(e=26.20)$ & $02(e=4.78)$ \\
\hline $100 \%$ & $16(\mathrm{e}=13.33)$ & $00(\mathrm{e}=2.67)$ & $14(\mathrm{e}=13.52)$ & $02(e=2.48)$ \\
\hline
\end{tabular}

Table - 9 (Association between Total BLS knowledge, BLS training, BLS experience among Dental interns)

\begin{tabular}{|c|c|c|c|c|}
\hline $\begin{array}{c}\text { Dental interns } \\
\text { BLS knowledge } \\
\text { Scores Scored }\end{array}$ & $\begin{array}{c}\text { BLS Work shop./ } \\
\text { training Attended } \\
(\mathbf{n}=\mathbf{6})\end{array}$ & $\begin{array}{c}\text { BLS Work shop / } \\
\text { training not attended } \\
(\mathbf{n}=\mathbf{7 2})\end{array}$ & $\begin{array}{c}\text { BLS Performed / } \\
\text { BLS experience } \\
(\mathbf{n}=\mathbf{0})\end{array}$ & $\begin{array}{c}\text { BLS not performed } \\
\text { ( } \mathbf{n = 7 8 )}\end{array}$ \\
\hline DENTAL INTERNS: & $00(\mathrm{e}=0.615)$ & $08(\mathrm{e}=7.385)$ & 00 & 08 \\
\hline $0 \%$ & $06(\mathrm{e}=1.539)$ & $14(\mathrm{e}=18.46)$ & 00 & 20 \\
\hline $25 \%$ & $00(\mathrm{e}=3.154)$ & $41(\mathrm{e}=37.84)$ & 00 & 41 \\
\hline $50 \%$ & $00(\mathrm{e}=0.692)$ & $09(\mathrm{e}=8.307)$ & 00 & 09 \\
\hline $75 \%$ & 00 & 00 & 00 & 00 \\
\hline $100 \%$ & \multicolumn{7}{r}{} & & $\mathrm{P}<0.05$ (significant)
\end{tabular}

Table - 10 (Contingency tables and Statistical correlations)

\begin{tabular}{|c|c|c|c|l|}
\hline $\begin{array}{l}\text { Association of } \\
\text { parameters }\end{array}$ & $\begin{array}{c}\text { Critical value } \\
\text { Chi square }\end{array}$ & $\begin{array}{c}\text { Probability } \\
\text { under Ho }\end{array}$ & P value & Statistical inference \\
\hline $\begin{array}{c}* \text { BLS knowledge score of } \\
\text { medical interns \& BLS work } \\
\text { shop training }\end{array}$ & 9.48 & 9.488 & $\mathrm{P}<0.05$ & $\begin{array}{l}\text { There is an impact of } \\
\text { attending BLS work shop on } \\
\text { the scoring pattern among } \\
\text { M edical interns }\end{array}$ \\
\hline $\begin{array}{l}* \text { BLS knowledge score of } \\
\text { medical interns \& } \\
\text { performing BLS }\end{array}$ & 20.497 & 9.488 & $\mathrm{P}<0.05$ & $\begin{array}{l}\text { There is an impact of } \\
\text { performing BLS on the } \\
\text { scoring pattern among } \\
\text { M edical interns }\end{array}$ \\
\hline $\begin{array}{l}* \text { BLS knowledge score of } \\
\text { dental interns \& BLS work } \\
\text { shop training }\end{array}$ & 18.850 & 9.488 & $\mathrm{P}<0.05$ & $\begin{array}{l}\text { There is an impact of } \\
\text { attending BLS work shop on } \\
\text { scoring pattern among } \\
\text { Dental interns }\end{array}$ \\
\hline $\begin{array}{l}* \text { BLS rate knowledge among } \\
\text { medical interns \& } \\
\text { performing BLS }\end{array}$ & 5.4158 & 3.841 & $\mathrm{P}<0.05$ & $\begin{array}{l}\text { Performing BLS has } \\
\text { influence on rate knowledge } \\
\text { among M edical interns }\end{array}$ \\
\hline $\begin{array}{l}* \text { BLS rate knowledge among } \\
\text { medical interns \& BLS } \\
\text { work shop training }\end{array}$ & 5.4158 & 3.841 & $\mathrm{P}<0.05$ & $\begin{array}{l}\text { Attending BLS work shop has } \\
\text { influence on rate knowledge } \\
\text { among M edical interns }\end{array}$ \\
\hline $\begin{array}{l}* \text { BLS ratio knowledge among } \\
\text { medical interns \& } \\
\text { performing BLS }\end{array}$ & 0.4556 & 3.841 & $\mathrm{P}<0.05$ & $\begin{array}{l}\text { Performing BLS has no } \\
\text { impact on BLS ratio } \\
\text { knowledge among M edical } \\
\text { interns }\end{array}$ \\
\hline $\begin{array}{l}* \text { BLS ratio knowledge among } \\
\text { medical interns \& BLS work } \\
\text { shop training }\end{array}$ & 0.8918 & 3.841 & $\mathrm{P}<0.05$ & $\begin{array}{l}\text { BLS work shop training has no } \\
\text { impact on BLS ratio knowledge } \\
\text { among M edical interns }\end{array}$ \\
\hline
\end{tabular}


Table - 10 ( continued)

\begin{tabular}{|l|c|c|c|l|}
\hline $\begin{array}{l}\text { Association of } \\
\text { parameters }\end{array}$ & $\begin{array}{c}\text { Critical value } \\
\text { Chi square }\end{array}$ & $\begin{array}{c}\text { Probability } \\
\text { under Ho }\end{array}$ & P value & Statistical inference \\
\hline $\begin{array}{c}\text { * Location knowledge of BLS } \\
\text { among medical interns \& } \\
\text { performing BLS }\end{array}$ & 13.316 & 3.841 & $\mathrm{P}<0.05$ & $\begin{array}{l}\text { Performing BLS has influence } \\
\text { on BLS location knowledge } \\
\text { among M edical interns }\end{array}$ \\
\hline $\begin{array}{c}\text { * Location knowledge of BLS } \\
\text { among medical interns \& } \\
\text { BLS work shop training }\end{array}$ & 4.866 & 3.841 & $\mathrm{P}<0.05$ & $\begin{array}{l}\text { Attending BLS work shop } \\
\text { has influence on BLS } \\
\text { location knowledge among } \\
\text { M edical interns }\end{array}$ \\
\hline $\begin{array}{l}\text { * BLS sequence knowledge } \\
\text { among medical interns \& } \\
\text { performing BLS }\end{array}$ & 0.757 & 3.841 & $\mathrm{P}<0.05$ & $\begin{array}{l}\text { Performing BLS has no } \\
\text { influence on BLS sequence } \\
\text { knowledge among M edical } \\
\text { interns }\end{array}$ \\
\hline $\begin{array}{l}\text { * BLS sequence knowledge } \\
\text { among medical interns \& } \\
\text { BLS work shop training }\end{array}$ & 2.457 & 3.841 & $\mathrm{P}<0.05$ & $\begin{array}{l}\text { BLS work shop training has no } \\
\text { influence on BLS sequence } \\
\text { knowledge among medical } \\
\text { interns }\end{array}$ \\
\hline
\end{tabular}

\section{Summary of Results:}

The summary of the results drawn from the above tables were asfollows:

1. The present study encompassed 84 interns from medical stream and 78 interns from Dental stream. (Table-1)

2. Female interns out numbered male interns in both medical and dental streams (Table - 1 )

3. There was no significant age difference between male \& female medical and dental interns. (Table - 1)

4. All the interns (100\%) from both medical and dental streams were aware of the BLS thus demonstrating a high level of awareness. (Table - 2)

5. All the interns (100\%) from both medical and dental streams were aware of the need and usefulness of possessing BLSknowledge. (Table - 2)

6. All the interns ( $100 \%)$ from medical and dental streams favored inclusion of BLS in their academic curriculum, thus stressing the need for a structured BLS training. (Table - 2)

7. M ajority of interns ( $87 \%$ medical, $81 \%$ dental) correctly knew that BLS could be performed at both with in and out side the hospital set ups. (Table - 3)

8. $98 \%$ of medical interns and $53 \%$ of dental interns had observed BLS being performed, suggesting medical interns were more fortunate than dental interns. (Table-3)
9. $85 \%$ medical interns had actually administered BLS in a real life situation while none ( $0 \%$ ) of the dental interns had performed the same, indicating poor hands-on BLS experience among dental interns. (Table - 3)

$10.85 \%$ of medical interns had received BLS training through BLS workshops while it was only $8 \%$ among dental interns, thus suggesting poor exposure to BLS training among dental interns. (Table-3)

11. Level of BLS knowledge was better among medical interns as they scored better than dental interns as evidenced by 37 (44\%) of medical interns, 69 ( $88 \%$ ) of dental interns had scored less than $50 \%$ of scores.(Table-5)

$12.62 \%$ medical and $50 \%$ dental interns self graded themselves to have average knowledge of BLS in a five level grading system. On comparing the scores scored by interns (on knowledge of BLS) and their own self grading of BLS knowledge, it was evident that interns demonstrated realistic assessment of themselves. (Table-5)

13. Non availability of professional training was quoted as the main reason for lack of BLS knowledge by maximum interns ( $67 \%$ medical \& $75 \%$ dental interns). A small percentage of interns ( $11 \%$ medical $\& 9 \%$ dental) quoted lack of interest also as the cause for the same. This shows an astonishingly similar trend in the 
perception of both medical \& dental streams of interns for not having an adequate BLS knowledge. (Table - 5)

14. Inferential statistical analysis reveled a clear association $(P<0.05)$ of better BLS Knowledge score $\&$ individual BLS knowledge components among the interns who had attended the BLS work shop and who had performed BLS. This makes it evident that the resuscitation experience (performing BLS) and training (attending BLSWork shop) would boost the confidence among interns. Table- 4, 6,7,8,9, 10)

Figure 1

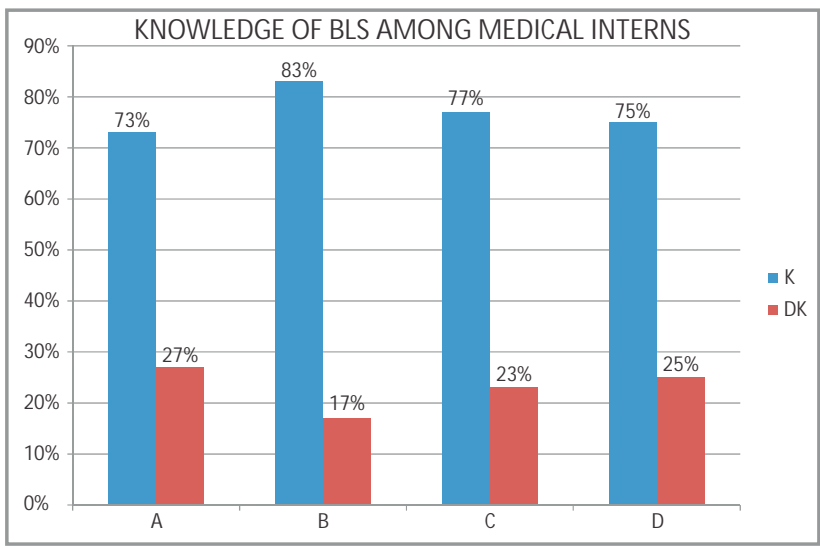

Figure -2

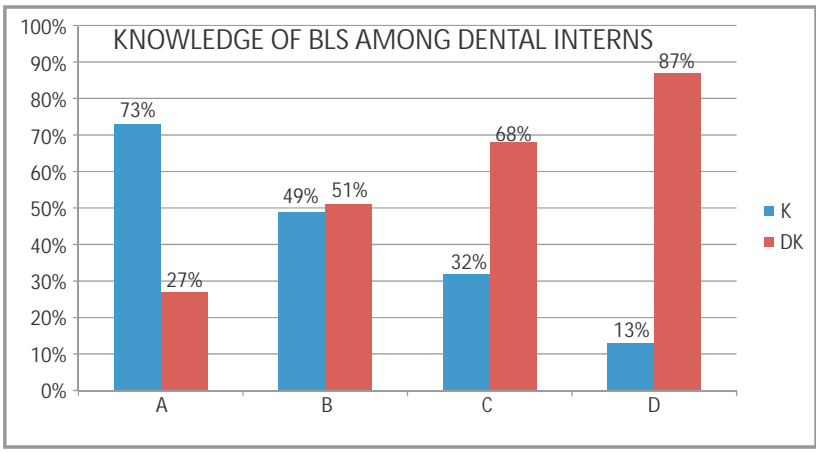

FOR FIGURES - $1 \& 2$ :

$A=$ Knowledge of Rate of cardiac compression during BLS delivery.

$B=$ Knowledge of Ratio of cardiac compressions to breath during BLS delivery.

$\mathrm{C}=$ Knowledge of Location for cardiac compression during BLS Delivery.

$D=$ Knowledge of Sequence of BLS components during BLS delivery.

$\mathrm{K}=$ Knows

DK =Does not know.
Figure -3



FOR FIGURE - 3 :

$\mathrm{E}=\mathrm{BLS}$ score of $50 \%$ and above.

$\mathrm{F}=\mathrm{BLS}$ score of below $50 \%$.

$M D=$ Medical interns.

$\mathrm{DN}=$ Dental interns.

\section{Discussion :}

The present study being an unique study analyzed the knowledge, awareness and attitude towards BLS among both medical and dental interns right at the point of completion of their internship. The results from the present study revealed the following:

1. It was most common to note Average knowledge of BLS among both medical and dental interns.

2. Medical interns were better than dental interns with respect to $B L S k n o w l e d g e$.

3. Level of BLS knowledge was not satisfactory especially in those who had not received any prior training(BLSwork shop).

4. Lack of professional training of BLS was regarded as the most common hindering factor responsible for poor BLSknowledge by interns.

5. Both medical and dental interns demonstrated realistic assesment of themselves while grading themselves about their knowledge of BLS.

6. Structured training of BLS was strongly favoured by all interns.

\section{Conclusions:}

From the present study we conclude the following:

1. Performing BLS and Attending BLS work shop plays a vital role in attaining BLS knowledge by both medical and dental interns. 
2. There is an absolute clear need for review of Basic Life Support (BLS) education for

3. There is a need for structured training of BLS and BLS must be included in the Medical and Dental academic curriculum. This will go a long way in improving the outcome of BLS delivery by interns (the future health care providers), thus immensely benefitting the society and also boosting the morale of the interns. Interns from both Medical and Dental streams.

\section{(Questionnaire)}

ADULT BASIC LIFE SUPPORT AWARENESS AND KNOWLEDGE IN INTERNS COM PLETING INTERNSHIP FROM DEEMED UNIVERSITY

\section{SLNO. : \\ AGE : \\ GENDER: Male / Female \\ COURSE : MBBS/ BDS}

1. Have you heard about BLS?

Yes/ No

2. Expand BLS

3. Do you think that all interns need to know about BLS? Yes/No

4. Do you think BLS training should be part of your medical curriculum? Yes/No

5. Do you think BLS should be done only in hospital settings?

Yes/No

6. Please indicate the reason for lack of knowledge about BLS?

a. Busy curriculum

b. Lack of interest

c. No professional training available

7. Have you heard about BTLS

Yes/No

If yes, Expand

8. Have you ever seen a BLS (CPR) being done? Yes/No

9. Have you ever done a BLS (CPR) on a patient? Yes/No

10. Have you ever attended a workshop on BLS? Yes/No

11. Indicate the number of compressions to be done in one minute in an adult?
a. 100
b. 200
c. 50
d. 30

12. Mark the ratio of chest compression to breath in adults?
a. $15: 2$
b. $3: 2$
c. $30: 2$
d. $100: 2$

13. Please indicate the location for chest compressions?

a. Left side of the chest

b. Right side of the chest

c. M id chest

d. Xiphisternum

14. Arrange them in orderly sequence.
a. Head tilt
b. Chin lift
c. Check pulse

15. Expand AED

16. Have you heard of Heimlich maneuver? Yes/No

17. Please indicate the dialing number for help in case of a medical emergency in your setup?
a. 911
b. 102
c. 104
d. 108

18. Please rate yourself on BLS knowledge?
a. Poor
b.Below average
c. Average
d.Good
e. Excellent

19. Is it useful to know about BLS? Yes/No

\section{References :}

1. Ritter G, Wolfe RA, Goldstein S et al : "The effect of by stander CPR on survival of out of hospital cardiac arrest victms". Am HeartJ 1985; 110: 932-7

2. Chandrasekaran S, Kumar S, Bhat SA et al : "Awareness of basic life support among medical, dental, nursing students \& doctors".Indian J Anaesth 2010; 54 : 121-6

3. SRaghavan, H Schneider, WGJ Kloeck: “Basic resuscitation-knowledge $\&$ skills of full time medical practitioners at public hospitals in northern province". SAMJ M ay 2000, 90(5):504-508

4. Hassan Zaheer, Zeba Haque : "Awareness about BLS(CPR) among medical students-status and requirements".JPM A Jan 2009; 59 : 1, 5759

5. PSPhillips, JP Nolan : "Training in basic and advanced life support in U Kmedical schools: Questionnaire survey". BMJ 2001 July 7; 323 (7303) $: 22-23$

6. Ji Ung Na, Min Soeb Sim, IK Joon Jo et al: "Emergency Med J doi : 10.1136 / Emer med - 2011 - 200633, Original article published on line Nov 2011 